\title{
II «sistema» della memoria in Amarcord di Federico Fellini
}

Giovanni Albertocchi

II presente contributo a questo seminario di aggiornamento intende proporre una lettura della cinematografia di Fellini in chiave letteraria. $\mathrm{Ho}$ o scelto una sezione assai importante, quella legata ai ricordi e quindi all'autobiografia: analizzandone le sequenze più significative, ho l'intenzione di far risaltare il «sistema» adottato dal regista nell'elaborare la sua poetica della memoria. Si tratta infatti di un «sistema» vero e proprio, che dispone delle sue leggi, di una tecnica e di una propria estetica.

Comincerò con il precisare, anche se non ce ne sarebbe bisogno, che I'autobiografia costituisce in genere la risorsa principale di chiunque si avventuri nel terreno della creazione. $\mathrm{N}$ el caso di Fellini, possiamo dire che ne ha fatto uso fino alla sazietà. Anche se, come manipolatore recidivo della propria esistenza, il regista appare più legato ad una disciplina letteraria che ad una esclusivamente cinematografica. Lungi da me l'idea di stabilire una frontiera fra i due generi: la promiscuità, se usata a fin di bene, è un'ottima opportunità espressiva. Vorrei soltanto far rilevare che Fellini, quando l'ispirazione lo spingeva verso la sua città natale (Rimini) o verso quella adottiva (Roma), componeva le immagini secondo tecniche e statuti assai più vicini alla letteratura che al cinema. La scrittura ha avuto del resto un ruolo determinante nella formazione del regista, dai racconti degli anni giovanili fino alle sceneggiature, tra cui ricorderò quella di Roma città aperta, scritta in collaborazione con Sergio Amidei, e di Paisà.

II viaggio di Fellini verso Rimini inizia nello stesso istante in cui la lascia, come M oraldo, il protagonista de I vitelloni. Tutti ricorderanno la bellissima sequenza in cui $M$ oraldo dal finestrino del treno che sta prendendo velocità «vede» nell' intimità delle diverse camere da letto gli amici assopiti nel loro torpore provinciale. Gli «interni» immaginati oscillano come se fossero anch'essi issati sul treno, suggerendo con grande efficacia la sensazione di uno «scarto» che sta per prendere il sopravvento sulla realtà. Ė, a mio avviso, lo «scarto» della memoria che ha già iniziato a provvedere alla sistemazione poe tica del passato. M oraldo, in partenza, si trova già in condizione di «ricordare» gli amici che sta lasciando. Sono le prime avvisaglie di un sistema che, come ho detto poc'anzi, appartiene all'ambito della letteratura. La memoria deve allontanarsi dai luoghi dell'esistenza per ricostruirli mediante la fantasia ed il ricordo. II recupero di Rimini si realizza quindi attraverso le leggi con- 
trapposte dell'oblio: è un luogo confuso, a metà strada tra la memoria che lo riscatta e l'oblio che lo sopprime.

Dalla genericità espressiva dei primi film, Rimini si dilata progressivamente fino a divenire, in Amarcord, la patria ideale dei ricordi e della creazione. II titolo, che in dialetto emiliano significa «mi ricordo», indica effettivamente la conclusione di un processo creativo che ha fatto del passato e della memoria il terreno privilegiato di ricerca. 0 gni film precedente è un semplice frammento, un ricordo parziale, che soltanto in Amarcord il regista riesce ad integrare nella poetica unitaria della memoria.

La strategia narrativa di Fellini, a differenza di altri autori che hanno «coniugato» la memoria secondo tempi intermittenti o circolari (vedi ad es. la definizione di «tempo curvo» usata da Cesare Segre per G abriel García M árquez), ${ }^{1}$ è invece lineare e strutturata sulla iterazione di una serie di temi biografici come la famiglia, la donna, la scuola, inseriti nei rispettivi contesti geografici. L 'aspetto iterativo non è casuale, ma corrisponde ad una tecnica di lavoro, a mio avviso, squisitamente letteraria. Fellini ci appare infatti nelle vesti di un filologo che lavora sulle varianti per giungere alla redazione finale del testo. Le varianti sono i frammenti progressivi della memoria ed i diversi film che le contengono, ed il testo è il passato del regista. Fellini compone quindi film-varianti che confluiranno nell'edizione critica del proprio passato cioè in Amarcord.

Come esempio di questo lavoro sulle varianti, possiamo utilizzare il personaggio del padre, qui come altrove, luogo emblematico di conflitti ed allo stesso tempo luogo privilegiato della memoria. D opo una serie di approcci a tale personaggio realizzati ne I Vitelloni (1953), la tipologia si definisce con maggior consistenza nelle esperienze successive:

1. La dolce vita (1959): il padre riemerge dall'assenza («non ci vediamo mai ») e dalla provincia, facendo una visita insperata, a Roma, al figlio $\mathrm{M}$ arcello. Costui vorrebbe saperne di più sul proprio genitore e fa delle avance in questo senso; ma riesce solo a conoscere qualche lato esterno del carattere, spigliato e galante, del genitore. II malessere dopo una serata «brava» riporta il padre lontano, nella vecchiaia e nella remota provincia.

2. Fellini 8 e 1/2 (1963): si perfeziona il tema del «padre assente». In una sequenza di sapore onirico, vediamo il figlio ( $M$ arcello M astroianni) che cerca di stabilire un dialogo con il padre («abbiamo parlato cosi poco») che invece si sottrae, scomparendo in un sentiero sotterraneo che potrebbe condurre all'al di là.

3. Roma (1971): qui la tipologia del padre si fa di «carne ed ossa». Appare la variante del padre irascibile. In una sequenza vediamo infatti il padre del protagonista-narratore (Fellini-giovane) che è preda di un attacco di rabbia mentre alla radio trasmettono una benedizione del Santo Padre. 
4. Amarcord (1973): si prosegue con gli effetti di irascibilità in «carne ed ossa», con la differenza, però, che tale manifestazione si esplica in un contesto narrativo di maggior portata e di maggior qualità letteraria. Si passa cioè da una serie di varianti paterne approssimative ad un personaggio completo, a tutto tondo. Sono diverse le ragioni che mandano su tutte le furie il padre di Titta, ed ognuna legata ad un diverso filo narrativo: c'è innanzitutto la situazione politica, particolarmente pesante per lui che è anarchico, poi il cognato fascista e scroccone che vive alle sue spalle e per finire il figlio T itta che ne combina di tutti i colori. La situazione esplode durante la sequenza in cui appare tutta la famiglia riunita a tavola, momento in cui confluiscono i tre fili narrativi. Mentre il brav'uomo osserva stizzito il cognato che mangia a sbafo e per di più sotto lo sguardo compiacente della sorella (la di lui moglie), suonano alla porta. II padre si alza, apre e viene a sapere che il figlio maggiore, T itta, al cinema Fulgor, ha orinato dalla galleria su uno spettatore della platea. II padre va in bestia, mentre il cognato, impassibile, chiede ancora dello stufato.

Si può dire che attraverso questa lunga sequenza (23 minuti) il personaggio del padre evolve in quello di capo-famiglia. «Poche volte, - sostiene T ullio Kezich - forse soltanto in certe commedie di Eduardo, la famiglia-tribù italiana è stata inquadrata con tanta amorosa e vulnerabile attenzione».2

Altro percorso della memoria è quello che riguarda il sesso, o meglio I'alone mitico in cui emerge una tipologia di donna ancestrale, a metà strada tra il demone ed il mostro. Anche qui si tratta di varianti che procedono verso un climax narrativo di più ampia portata. Si parte dalla «saraghina» di Fellini 8 e 1/2 per arrivare all' opulenza espressiva della «tabaccaia» di Amarcord. Ancora una volta, il film dei ricordi funge da edizione critica della memoria. O ffro di seguito lo schema delle diverse varianti:

1. Fellini 8 e 1/2: appare la «saraghina», un donnone cencioso che con le sue danze procaci, a pagamento, sulla spiaggia, turba la fantasia dei collegiali. I Padri del collegio la considerano emissaria diretta di Satana ed operano di conseguenza, sottoponendo Fellini-bambino ad un lavaggio del cervello. M a il collegiale torna recidivo ad ammirare la donna sulla spiaggia, ormai conquistato dal mistero e dalla trasgressione. La «saraghina», ha dichiarato Fellini nel libro-intervista curato da Giovanni Grazzini, «è la prima prostituta che ho visto nella mia vita [... ] Con due soldi ci faceva vedere in silenzio il sedere che copriva tutto il cielo». 3

2. Amarcord: la «saraghina» evolve nel ricordo fino a dar vita a due personaggi diversi, pur appartenenti alla stessa tipologia, la «volpina» che turba con le sue profferte e la sua ancestrale insaziabilità le maestranze al lavoro, e la «tabaccaia» dalle forme straripanti che eccita le fantasie erotiche di

3. Fellini (1983: 72). 
T itta. La «tabaccaia» riceve il testimone dalle colleghe che l'hanno preceduta in questo splendido affondo sul mito, offrendo, in un certo senso, la sequenza narrativa più completa e suggestiva.

Altro esempio di questo lavoro sulle varianti ci viene offerto dal trattamento riservato ai ricordi della scuola, ove si passa dall'approccio frammentario di Roma (la scolaresca che assiste alla proiezione di un «filmino» sulle bellezze della «città eterna») allo splendido capitolo di Amarcord, in cui Fellini riesce finalmente a scattare l'autentica «foto di gruppo» delle proprie memorie scolastiche.

Abbiamo visto sin qui la conformazione tecnica del sistema della memoria, basato sulla progressione delle varianti. M a il sistema prevede anche strategie più interne che riguardano i meccanismi del ricordo e della sua elaborazione o riproduzione artistica.

Al centro del ricordo c'è naturalmente il vissuto di Fellini che viene però manipolato da agenti estranei, come la menzogna o, per usare una categoria più appropriata alla creazione artistica, la finzione. È lo stesso Fellini a dichiarare che i suoi film:

Sono completamente inventati, dalla prima all' ultima scena: non discendono dalla realtà. La Rimini alla quale sono affezionato, I'ho ripetuto fino alla nausea, è quella che ho ricostruito in studio, l'unica vera. 4

La città natale del regista ha bisogno dei fondali dipinti e del mare di plastica per affermare la propria autenticità.

Se si vuole testimoniare la verità, - aggiunge Fellini - dire «jo» è già un punto di vista sbagliato. Per dire una cosa vera bisognerebbe togliere «io»; e invece non si può farne a men 0.5

II ricordo tradito dall'immaginazione è quello che più si avvicina alla verità, in quanto riflesso di un patrimonio collettivo in cui si devono riconoscere Fellini e gli spettatori. Fellini «mente» per poter narrare una verità collettiva, per poter ricordare come singolo ed allo stesso tempo come membro della grande comunità degli adulti che hanno un passato comune alle spalle. Secondo il regista, il cinema è infatti rappresentazione dell'inconscio collettivo:

La produzione artistica, cioè, non sarebbe al tro che l'attività onirica dell'umanità; il pittore, il poeta, il romanziere e anche il regista, risponderebbero a questa funzione, di elaborare, organizzare col proprio talento i contenuti dell' inconscio collettivo, esprimendoli, rivelandoli sulla pagina, sulla tela o sullo schermo. ${ }^{6}$

4. Kezich (1988: 5).

5. Ibid.

6. Fellini (1983: 73). 
L'approccio al passato deve quindi avvenire cercando di tenere a bada l'io che vorrebbe dire la sua. La menzogna è quindi una sorta di antidoto al rischio di veder affondare la propria creazione nel mare della soggettività. Ed è inoltre il correlativo oggettivo di un passato che non può che essere narrato con gli opportuni aggiustamenti.

L'artista che mente è anche, è bene ricordare, l'artista che crea, il demiurgo che opera nel territorio misterioso della finzione:

II cinema è un modo divino di raccontare la vita, di far concorrenza al padreterno! $\mathrm{N}$ essun altro mestiere consente di creare un mondo che assomiglia cos̀ da vicino a quello che conosci, ma anche agli altri sconosciuti, paralleli, concentrici. Per me il posto ideale, I'ho già detto tante volte, è il Teatro 5 di Cinecittà, vuoto. Ecco, l'emozione assoluta, da brivido, da estasi, è quella che provo di fronte al teatro vuoto: uno spazio da riempire, un mondo da creare. $\mathrm{H}$ o la totale presunzione di essere un demiurgo. ${ }^{7}$

\section{Bibliografia}

FelLIN I, F. (1983). Intervista sul cinema (a cura di G G Gazzini). Bari: Laterza.

KEZICH, T . (1988). Fellini. Milano: Rizzoli.

Segre, C. (1969). I segui ela critica. Torino: Einaudi.

7. Ibid.: 83. 